therefore may not have been a homogeneous group, and indeed the variation which was clearly evident in the Australians and Tasmanians during the nineteenth century, before decimation by European disease and "administration", gives support to the idea of a heterogeneous mosaic in these populations.

As far as Kow Swamp is concerned, anthropologists will look forward to publication of a detailed report and to the availability of casts for comparative study. For the present the biological status of this prehistoric Australian group will remain somewhat controversial.-From a Correspondent.

\section{POLYSACCHARIDES}

\section{The Forgotien Molecules}

from our Molecular Biology Correspondent IN an era in which biochemistry, for better or worse, is dominated by macromolecules, the polysaccharides have been more or less lost to view. Most biochemists would probably admit if pressed that they regard their colleagues in the carbohydrate field as a race apart, plying their lugubrious trade in dignified isolation, far from the bustle of molecular biology. Of late Rees and his associates have done much to narrow the gulf by showing that some at least of the polysaccharides possess an unsuspected conformational versatility in solution, and are capable of interactions and transitions that can be described in language comprehensible to those who have grown up with nucleic acids and proteins.

One knows, of course, from X-ray studies that ordered structures exist in the solid state, but in solution the evidence is circumstantial. In polymers like the carrageenans and agarose, gelation is accompanied by optical changes. In the case of $\kappa$-carrageenan, in particular, these have been interpreted in terms of the conversion of randomly coiled chains into interrupted double helices. From a number of physical and chemical lines of evidence, Rees and his colleagues inferred that the non-covalent cross-links, which are required for gel formation, depend for their existence on the presence of helix-interrupting kinks in the chain. These kinks have been chemically characterized, and are subject to specific hydrolytic scission. The resulting fragments undergo the physical changes normally associated with gelation, without, however, generating any gel. In particular the order-disorder transition is accompanied by the two-fold change in molecular weight, which is expected to result from strand separation, when the double helices melt. The double helix, it may be noted, is also the structure deduced from X-ray analysis of fibrous $k$-carrageenan.

Now Dea, McKinnon and Rees (J. Mol. Biol., 68, 153; 1972) have shown that specific conformation-linked interactions can occur in solution between polysaccharides of different kinds. Agarose, like the carrageenans, displays a change in optical rotation, betokening the breakdown of an ordered structure, when gel melts, and this effect is fully reversible, though with a huge hysteretic lag, amounting to some $50^{\circ}$. The galactomannans, on the other hand, do not gel, and show no optical transitions. These molecules consist of a mannan backbone, with single galactose units grafted on at intervals, the tendency being for the galactose-substituted residues to occur in blocks. In the solid state these polymers are extended chains, and so, Dea et al. suggest, are they in solution. When mixed with the nongelling fragmented $k$-carrageenan the gelling capacity reappears.

The same phenomenon is observed in mixtures of the galactomannan with fragmented agarose, and the galactomannan will also provoke gelation in solutions containing intact agarose at concentrations below that at which it will gel by itself. The greater the density of galactose substitution in the galactomannan the lower is its ability to form such mixed gels. The formation of helices in the mixtures is inferred both from the changes in optical rotation and the behaviour of the dye, methylene blue, which acts as an empirical indicator of conformation: it evidently interacts with the ordered, presumed helical, state of the polysaccharide, and this inter- action elicits large changes, characteristic of the formation of dye stacks, in the visible absorption spectrum, as well as the onset of induced Cotton effects in the intrinsically optically inactive chromophore.

Dea et al. have a model for the interaction of the polymers. On the grounds that galactose substitution inhibits association with the helices of carrageenan or agarose, it is postulated that it is the blocks of unsubstituted residues that combine in extended form with the helices, the uncombined galactose-rich segments then functioning as cross-links. In the agarose complex the optical rotation undergoes a change in the course of gelation, which is in the opposite sense to that associated with the coil $\rightarrow$ helix transition of agarose alone. This suggests that there is an overriding contribution of the opposite sign from a conformational change in the galactomannan. This interpretation is borne out by re-heating experiments, in which the very large hysteresis that characterizes the agarose transition allows a partial separation of the contributions of the complex and of the uncomplexed agarose. Rees has formulated empirical rules which relate optical rotation with backbone torsional angles, taking into account restrictions imposed by model building. Application of these rules to the contribution of the galactomannan in the complex structure permits an enlightened guess at its possible conformation in this environment.

Dea et al. offer finally some cogitations on what they conceive to be the biological relevance of these interactions. The unsubstituted parts of the galactomannan they regard as a model for the rather similar structural poly-

\title{
Stratospheric Warmings in the Southern Hemisphere
}

STUDiEs of the southern hemisphere stratosphere may be aided as a result of an investigation carried out by Eric L. Unthank of the University of Melbourne and reported in next Monday's Nature Physical Science (August 14). Unthank has found that measurements taken at an observing station near Melbourne can give an indication of conditions in the stratosphere between Australia and Antarctica. This discovery is helpful, as one of the obstacles to a better understanding of the southern stratosphere is the sparsity of observing stations.

In particular Unthank is interested in those occasions known as stratospheric warmings, which occur when the circulation of the winter stratosphere is disrupted and the stratospheric temperature rises for several weeks by as much as several tens of degrees. $\mathrm{He}$ has compared observations of the stratosphere made from a station near Melbourne with observations taken 1,300 miles to the southeast at Macquarie Island.

The measurements show that the temperature of the stratosphere about $30 \mathrm{~km}$ above Macquarie Island correlates with the stratospheric winds at about $30 \mathrm{~km}$ above the observing station near Melbourne. When temperatures are low above Macquarie Island there are westerly winds above the mainland station, and high temperatures above Macquarie Island occur with easterly winds above the mainland station.

This result is a step towards the reduction of the disparity between what is known of the northern and southern stratospheres. The extent of the correlation between the two widely separated stations should throw further light on the extent of strato. spheric warmings and associated effects in the southern hemisphere. 